rare i due concetti come equivalenti. Senonché nel discorso di Weber sono due tipi di democrazia non solo distinti, ma a ben guardare, contrapposti. Fuorviante può riuscire anche la sostituzione di «democrazia ordinata» con «democrazia regolata» in un'altra citazione ( $\mathrm{p}$. 174). Non di rado, poi, le citazioni sono accompagnate da rinvii bibliografici errati. Entrambe le sopra discusse citazioni di Weber, per esempio, non si trovano alla pagina indicata ma altrove nel testo. Una disattenzione minore, che però ostacola la lettura critica di questo provocatorio libro.

\title{
[Luciano Cavalli]
}

ALAN S. MILWARD, The European Rescue of the Nation-state, Londra, Routledge, 1992, pp. 477.

Alan Milward, che si era già segnalato con The reconstruction of Western Europe (1945-5), si ripropone con questo volume (nonché con The frontier of national sovereignty. History and theory 1945-92, Routledge, 1993 che arricchisce la teoria e l'analisi storica contenuta in questa opera) come lo studioso realista della Comunità europea, in piena contrapposizione non solo con i teorici funzionalisti dell'integrazione, ma anche con la cosiddetta corrente istituzionalista delle relazioni internazionali.

Milward si preoccupa di smentire il postulato degli istituzionalisti secondo i quali nella misura in cui la Comunità si afferma, a perdere terreno sarà lo stato-nazione. Egli infatti sostiene che la Comunità è parte integrante della riaffermazione dello stato-nazione in Europa. Tale istituzione infatti, crollata durante la seconda guerra mondiale, ha saputo recuperare il consenso attraverso l'assicurazione del benessere e della crescita economica. Tale sforzo è stato compiuto in parte grazie alla promozione di politiche nazionali, in parte grazie alla costituzione di una nuova organizzazione politica (prima la CECA, poi la $\mathrm{CEE}$ ), su cui gli stati continuano però ad esercitare il proprio controllo.

Milward ridimensiona l'attribuzione di sovranazionalità all'Alta autorità della CECA: laddove si è realizzata, essa è servita ai governi per far accettare ai propri cittadini misure impopolari, quali ad esempio la ristrutturazione e la chiusura di fabbriche. Le decisioni formulate da tale organo si sono sempre configurate come il risultato dell'accordo tra i sei governi; in caso di disaccordo, è sempre mancata la possibilità di formulare una politica coerente.

Nel corso dei primi decenni, dunque, la Comunità si è affermata sull'onda di un modello economico interventista all'interno (supporto dell'agricoltura, alti salari, modernizzazione industriale, promozione 
del welfare state) e protezionista all'esterno, nonostante i vari round del GATT. Negli anni '80 però, di fronte alla perdita di competitività della Comunità, soprattutto in relazione alle esportazioni giapponesi, $\mathrm{i}$ vari governi hanno abbracciato una nuova ideologia fondata sul liberismo, auspicando il rilancio dei settori produttivi grazie alla deregolazione interna. La conseguenza di tutto ciò è stato l'Atto unico europeo e la scadenza del 1992 per eliminare le barriere non tariffarie tra i paesi membri. Come negli anni ' 50 , la responsabilità comunitaria di politiche impopolari evita crisi nazionali di consenso.

Il pensiero di Milward si contrappone anche alla classica spiegazione di tipo politico data dagli studiosi al processo di formazione della Comunità: il collective binding della Germania. Tale fattore è giudicato secondario: secondo l'A., sono state le motivazioni economiche a convincere il governo francese a promuovere l'alleanza con la Germania, paese che rappresentava il mito della crescita economica basata sull'esportazione e sulla modernizzazione industriale. Il Regno Unito, secondo Milward, perse un'opportunità decisiva rifiutando di entrare nella Comunità. L'orgoglio nazionale di avere una sterlina forte portò il governo inglese a mantenere tassi di interesse alti, grazie anche all'azione di lobby della City londinese. Il mito della comunità anglofona (il Commonwealth) e dell'alleanza privilegiata con gli Stati Uniti, risultato anche di un certo disprezzo per le potenze europee sconfitte in guerra, offuscò i governanti inglesi, al punto di non renderli coscienti che il mercato europeo e le politiche europee potevano rappresentare un'ancora di salvezza per l'industria inglese, che invece non fu modernizzata, fu penalizzata dall'aggiustamento e surclassata dall'industria tedesca.

Secondo Milward dunque la Comunità non è un'altra forma di governo, non ha vita indipendente: al contrario è del tutto compatibile con gli interessi degli stati-nazione. Tali attori dunque si servono ora dello strumento Comunità (opzione integrazione), ora dello strumento governativo (opzione interdipendenza): la scelta dipende dalla natura delle politiche da attuare. Milward ammette che la sua teoria non ha grosse capacità previsionali: si limita ad affermare che l'integrazione andrà avanti nella misura in cui le circostanze del periodo 1945-68 si ripeteranno. Allora la modernizzazione industriale e il protezionismo commerciale servirono da collante. Negli anni '70 lo shock petrolifero spinse i governi europei ad adottare il sistema monetario. Negli anni ' 90 è stato il timore della Germania unita a portare a Maastricht. Nel futuro, sembra che Comunità e stato-nazione continueranno ad essere compatibili, grazie alla formula dell'Europa a due velocità e dell'opting out (vedi i casi di Danimarca e Regno Unito).

La radicalità del pensiero di Milward, così contro-corrente ma nello stesso tempo convincente, solleverà critiche da parte degli studiosi istituzionalisti. In realtà, seppur il punto di arrivo è diverso - 
l'autonomia o non dell'attore Comunità -, il presupposto di partenza è lo stesso: quello cioè secondo cui i governi sono obbligati ad utilizzare uno strumento diverso dalle politiche nazionali, per raggiungere obiettivi che altrimenti non sarebbero capaci di realizzare. In ogni caso, il periodo attuale rappresenta una congiuntura favorevole - la futura integrazione monetaria sembra tramontare di fronte ai diversi orientamenti di politica economica dei singoli governi - per sostenere che la Comunità è controllata dagli stati-nazione.

[Fabio Fossati]

GIANFRANCO PASQUiNo (a cura di), Votare un solo candidato. Le conseguenze politiche della preferenza unica, Bologna, Il Mulino, 1993, pp. 360.

Questa antologia di ricerche empiriche dedicata agli effetti dell'adozione della preferenza unica nelle elezioni italiane del ' 92 potrebbe, a prima vista, sembrare una sfortunata iniziativa editoriale.

Quanti dei nomi che si succedono nelle tabelle e nelle analisi di Ceccanti, Radaelli, Feltrin, Pellicani, Anderlini, Massari e Vassallo avranno ancora gli onori della cronaca politica? E a quanti potrà ancora interessare una riforma i cui effetti resteranno per sempre confinati ad un'unica consultazione elettorale?

Paradossalmente, proprio la sua inattualità, così insolita in un libro tanto recente, consente di apprezzarne le qualità durature. Nelle statistiche sui voti e nei resoconti quasi giornalistici, domande con un respiro teorico generale trovano una parziale, sebbene provvisoria, risposta. Ė lo stesso Pasquino ad enunciarle nell'introduzione: seguendo la falsa riga dell'ormai celebre pamphlet di Hirschman sulle retoriche della reazione, Pasquino reinterpreta le critiche degli oppositori alla preferenza unica come altrettanti argomenti a favore della inutilità, perversità e pericolosità delle riforme elettorali. Le ricerche condotte su cinque circoscrizioni - a vario titolo significative (le due maggiori, una tradizionalmente rossa, una tradizionalmente bianca ed una meridionale) - rispondono a tali critiche e offrono un'occasione interessante per riflettere sulle capacità di apprendimento delle nuove regole da parte dei singoli candidati, specie se paragonate alle performances spesso scadenti dei rispettivi partiti.

Il resoconto di Ceccanti sull'andamento complessivo delle elezioni politiche del '92, confrontato con quello delle elezioni dell' 87 e le ricerche circostanziate di Radaelli per la circoscrizione Milano-Pavia e di Massari per Roma-Viterbo-Latina-Frosinone confutano la tesi della futilità, secondo cui la preferenza unica non avrebbe avuto alcuna conseguenza significativa. La contrazione del mercato delle preferenze 